Aquat. Living Resour., 1996, 9, 217-223

\title{
Modèles loglinéaires pour l'étude des associations entre captures par unité d'effort et variables hydroclimatiques
}

\author{
Jacques Badia ${ }^{(1)}$ et Patrick Prouzet \\ $(2)$ \\ (1) INRA, Unité de Biométrie el d'Intelligence artificielle, \\ B.P. 27, 31326 Castanet Tolosan Cedex, France. \\ (2) IFREMER/INRA, Station d'Hydrobiologie de Saint-Pée-sur-Nivelle, \\ B.P. 3, 64310 Ascain, France.
}

Reçu le 14 septembre 1995; accepté le 7 mars 1996.

Badia J., P. Pouzet. Aquat. Living Resour., 1996, 9, 217-223.

Loglinear models for studying associations between catches per unit effort and hydroclimatic variables.

Abstract

In this paper we study associations between categorical variables. We present an approach based on loglinear modelling. A better knowledge of the influence of the intensity of tidal coefficient $(\mathrm{cm})$ and of the rate of river flow (df) on catch per unit effort (cpue) of allis shad (Alosa alosa) was obtained. Analysis performed indicated that an increase of catch per unit effort is proportionally associated with the increase in the tidal coefficient. We also noted the influence of the rate of flow.

Keywords: Loglinear model, catch per unit effort, environmental variables, Alosa alosa, allis shad.

Résumé

Dans cet article, nous étudions les associations entre variables qualitatives. Nous présentons une approche basée sur l'utilisation du modèle loglinéaire. Appliqués aux associations entre captures par unité d'effort (cpue) d'aloses (Alosa alosa), coefficient de marée $(\mathrm{cm})$ et débit fluvial (df), différents modèles mettent en évidence que les captures par unité d'effort augmentent proportionnellement à la valeur du coefficient de marée et qu'elles ont des évolutions conjointes moins structurées avec le débit fluvial.

Mots-clés : Modèle loglinéaire, capture par unité d'effort, variables environnementales, Alosa alosa, grande alose ou alose vraie.

\section{INTRODUCTION}

Le modèle linéaire généralisé est un outil efficace d'analyse des données qualitatives. En particulier, lorsque des variables catégorielles sont construites à partir de variables continues, les modèles loglinéaires permettent de définir des mesures d'associations. Dans ce qui suit, nous nous intéressons essentiellement à la méthodologie statistique exposée dans de nombreux ouvrages tels que Bishop et al. (1975), Fienberg
(1980) et Agresti (1990), pour montrer la richesse de cette approche peu utilisée en hydrobiologie ou en halieutique. Nous présentons différentes modélisations décrivant les associations entre variables catégorielles, nous les illustrons par les données du tableau 1 qui se réfèrent aux résultats des pêches d'aloses (Alosa alosa) dans le fleuve Adour entre 1988 et 1991 (Prouzet et al., 1994). Chaque cellule de ce tableau contient le nombre d'observations pour chaque combinaison des modalités des variables captures par unité d'effort (cpue), débit 
Tableau 1. - Observations par combinaison des modalités des variables captures par unité d'effort, débit fluvial et coefficient de maréc. Observations for the combination of catch per unit effort, rate of flow and tidal coefficient variables.

\begin{tabular}{|c|c|c|c|c|c|}
\hline \multirow[t]{2}{*}{ Débit fluvial } & \multirow[t]{2}{*}{ Coefficient de marée } & \multicolumn{4}{|c|}{ Captures par unité d'effort } \\
\hline & & Faible & Faible à Moyen & Moyen à Fort & Fort \\
\hline Faible & $\begin{array}{l}\text { Faible } \\
\text { Faible à Moyen } \\
\text { Moyen à Fort } \\
\text { Fort }\end{array}$ & $\begin{array}{r}11 \\
4 \\
7 \\
5\end{array}$ & $\begin{array}{r}5 \\
11 \\
5 \\
1\end{array}$ & $\begin{array}{l}2 \\
4 \\
9 \\
5\end{array}$ & $\begin{array}{l}1 \\
2 \\
4 \\
4\end{array}$ \\
\hline Faible à Moyen & $\begin{array}{c}\text { Faible } \\
\text { Faible à Moyen } \\
\text { Moyen à Fort } \\
\text { Fort }\end{array}$ & $\begin{array}{l}4 \\
8 \\
4 \\
4\end{array}$ & $\begin{array}{l}4 \\
6 \\
3 \\
7\end{array}$ & $\begin{array}{l}7 \\
4 \\
6 \\
7\end{array}$ & $\begin{array}{l}5 \\
6 \\
8 \\
7\end{array}$ \\
\hline Moyen à Fort & $\begin{array}{l}\text { Faible } \\
\text { Faible à Moyen } \\
\text { Moyen à Fort } \\
\text { Fort }\end{array}$ & $\begin{array}{l}5 \\
3 \\
2 \\
3\end{array}$ & $\begin{array}{l}4 \\
8 \\
3 \\
4\end{array}$ & $\begin{array}{l}5 \\
4 \\
5 \\
9\end{array}$ & $\begin{array}{l}9 \\
6 \\
6 \\
7\end{array}$ \\
\hline Fort & $\begin{array}{l}\text { Faible } \\
\text { Faible à Moyen } \\
\text { Moyen à Fort } \\
\text { Fort }\end{array}$ & $\begin{array}{r}15 \\
9 \\
4 \\
7\end{array}$ & $\begin{array}{l}7 \\
4 \\
1 \\
6\end{array}$ & $\begin{array}{l}3 \\
2 \\
5 \\
2\end{array}$ & $\begin{array}{r}2 \\
3 \\
9 \\
10\end{array}$ \\
\hline
\end{tabular}

fluvial (df) et coefficient de marée $(\mathrm{cm})$. Dans une première partic, nous présentons brièvement le modèle loglinéaire standard pour des tableaux de contingences à deux et à trois dimensions. Dans une deuxième partie, nous analysons les tableaux marginaux $\mathrm{cm}$ et cpue (Tableau 2a) puis df et cpue (Tableau 2 b) pour illustrer l'introduction dans le modèle standard de la relation d'ordre entre les modalités des variables. Nous étudions ensuite le tableau complet (Tableau 1) qui résume les informations sur les captures et sur les conditions hydroclimatiques des pêches.

\section{MATÉRIELS ET MÉTHODES}

\section{Les observations}

On trouvera dans Prouzet et al., (1994), une description précise du protocole de recueil des données et des caractéristiques de la pêcherie. Très brièvement, les données analysées sont les captures moyennes journalières entre 1988 et 1991 relevées dans les carnets de pêche mis en place par le Comité Interprofessionnel des Poissons migrateurs et des Estuaires (CIPE) et deux paramètres physiques qui caractérisent le milieu aquatique : le coefficient de marée qui est un coefficient astronomique exprimé en centième dont la valeur peut, sur l'année, être comprise entre 20 pour les marées de morte-eau les plus faibles et 120 pour les marées de vive-eau d'équinoxe; le débit fluvial journalier exprimé en mètre cube par seconde, est mesuré dans des stations de jaugeage. Les découpages en classes ont été effectués selon les quartiles pour les cpue. Pour prendre en compte les variations annuelles de l'abondance, la répartition en classe de cpue a été faite par année. Pour les variables physiques $\mathrm{cm}$ et $\mathrm{df}$, le codage a été effectué d'après les histogrammes des valcurs, toutes années confondues.

\section{Les modèles}

Pour le Tableau 2, nous adoptons les notations suivantes : $i$ est l'indice de ligne, $j$ l'indice de colonne, $I(=1)$ le nombre de lignes et.$J(=1)$ le nombre de colonnes. Les $n(=342)$ observations de l'échantillon sont réparties dans les $N=I J(=16)$ cellules du tableau. L'effectif de chaque cellule $i j$ est $n_{i j}$, il y a $n_{i+}$ observations dans la ligne $i$ et $n_{+j}$ dans la colonne $j$.

Dans le cas d'un échantillon multinomial, les probabilités $\left\{\pi_{i j}\right\}$ des cellules $\{i j\}$ représentent la distribution conjointe des deux catégories de réponses. $\mathrm{Si}$ les observations sont indépendantes $\left(\pi_{i j}=\right.$ $\left.\pi_{i+} \pi_{+j}\right)$, les effectifs espérés $\left(m_{i j}=n \pi_{i j}\right)$ sont

Tableau 2. - Observations par combinaison des modalités des variables captures par unité d'effort: a) coefficient de marée, b) débit fluvial.

Observations for the combination of catch per unit effort: a) tidal coefficient variables, b) rate of flow variables.

Captures par unité d'effort

Faible Faible à Moyen Moyen à Fort Fort

a) Coefficient de marée Faible Faible à Moyen Moyen à Fort Fort

35
24
17
19

$$
\begin{aligned}
& 20 \\
& 29 \\
& 12 \\
& 18
\end{aligned}
$$

17

$14 \quad 17$

b) Débit fluvial Faible Faible à Moyen Moyen à Fort Fort

$\begin{array}{ll}27 & 22 \\ 20 & 20 \\ 13 & 19 \\ 35 & 18\end{array}$

17 17 27 28 
égaux à $m_{i j}=n \pi_{i+} \pi_{+j}$ pour tout $i$ et $j$. Sur l'échelle logarithmique, l'indépendance $s$ 'exprime sous la forme additive $\log m_{i j}=\log n+\log \pi_{i+}+\log \pi_{+j}$. En notant $X$ et $Y$ les variables ligne et colonne, l'équation précédente est équivalente à

$$
\log m_{i j}=\mu+\lambda_{i}^{X}+\lambda_{j}^{Y}
$$

avec $\mu$ le paramètre général, $\lambda_{i}^{X}$ le paramètre associé à la modalité $i$ de la variable $X$ et $\lambda_{j}^{Y}$ le paramètre associé à la modalité $j$ de la variable $Y$. Pour être identifiables, ces paramètres satisfont à des contraintes telles que $\sum_{i} \lambda_{i}^{X}=\sum_{j} \lambda_{j}^{Y}=0$.

S'il existe une dépendance entre les variables $X$ et $Y$, le modèle s'écrit

$$
\log m_{i j}=\mu+\lambda_{i}^{X}+\lambda_{j}^{Y}+\lambda_{i j}^{X Y}
$$

Les nouveaux paramètres $\lambda_{i j}^{X Y}$ mesurent les écarts à l'indépendance des variables $X$ et $Y$, ce sont des paramètres d'association. Pour être identifiables, ces paramètres satisfont aux contraintes $\sum_{i} \lambda_{i j}^{X}=$ $\sum_{j} \lambda_{i j}^{Y}=0$.

Considérons maintenant les observations du Tableau 1 relatives aux variables $X(d f)$ et $Y(\mathrm{~cm})$ croisées avec la variable $Z$ (cpue). Avec des notations analogues aux précédentes :

(i) si les trois variables sont mutuellement indépendantes $\left(\pi_{i j k}=\pi_{i++} \pi_{+j+} \pi_{++k}\right.$ pour tout $i, j$ et $k$ ), le modèle d'indépendance mutuelle s'écrit $\log m_{i j k}=\mu+\lambda_{i}^{X}+\lambda_{j}^{Y}+\lambda_{k}^{Z}$.

(ii) si la variable $Z$ est indépendante de $X$ et de $Y\left(\pi_{i j k}=\pi_{i j+} \pi_{++k}\right.$ pour tout $i, j$ et $\left.k\right)$, le modèle d'indépendance jointe s'écrit $\log m_{i j k}=$ $\mu+\lambda_{i}^{X}+\lambda_{j}^{Y}+\lambda_{k}^{Z}+\lambda_{i j}^{X Y}$.

(iii) si $X$ et $Y$ sont indépendantes dans le tableau partiel associé à la modalité $k$ de $Z, X$ et $Y$ sont dites conditionnellement indépendantes au niveau $k$ de $Z$. Comme $\pi_{i j \mid k}=\pi_{i+\mid k} \pi_{+j \mid k}$ pour tout $i$ et $j$ avec, $\pi_{i j \mid k}=\pi_{i j k} / \pi_{++k}$ la distribution conjointe de $X$ et $Y$ au niveau $k$ de $Z$, le modèle d'indépendance conditionnelle de $X$ et de $Y$ s'écrit :

$$
\log m_{i j k}=\mu+\lambda_{i}^{X}+\lambda_{j}^{Y}+\lambda_{k}^{Z}+\lambda_{i k}^{X Z}+\lambda_{j k}^{Y Z}
$$

Pour les tableaux à trois dimensions, $\log m_{i j k}=$ $\mu+\lambda_{i}^{X}+\lambda_{j}^{Y}+\lambda_{k}^{Z}+\lambda_{i j}^{X Y}+\lambda_{i k}^{X Z}+\lambda_{j k}^{Y Z}$ est le modèle loglinéaire le plus général qui peut être analysé. Pour l'interpréter, il faut décrire les associations $\lambda_{i j}^{X Y}, \lambda_{i k}^{X Z}$ et $\lambda_{j k}^{Y Z}$ qui sont des fonctions des rapports des chances (odds ratios) conditionnels (Agresti, 1990).
Dans le tableau marginal $(X \times Y)$, obtenu en sommant sur toutes les modalités $k$ de la variable $Z$, il y a $(I-1)(J-1)$ odds ratios marginaux

$$
\begin{aligned}
\theta_{i j}^{X Y}= & \frac{\pi_{i j+} \pi_{i+1, j+1,+}}{\pi_{i+1, j,+} \pi_{i, j+1,+}} \\
& (1 \leq i \leq I-1,1 \leq j \leq J-1)
\end{aligned}
$$

qui décrivent l'association marginale de $X$ et de $Y$.

Dans le tableau partiel associé à la modalité $k$ de la variable $Z$ il y a $(I-1)(J-1)$ odds ratios conditionnels

$$
\begin{aligned}
\theta_{i j(k)}= & \frac{\pi_{i j k} \pi_{i+1, j+1, k}}{\pi_{i, j+1, k} \pi_{i+1, j, k}} \\
& (1 \leq i \leq I-1,1 \leq j \leq J-1)
\end{aligned}
$$

qui décrivent l'association conditionnelle de $X$ et de $Y$. Ces odds ratios sont les mêmes si $Z$ et $X$ ou $Z$ et $Y$ sont conditionnellement indépendantes (Bishop, 1971). De même, les tableaux marginaux $(X \times Z)$ et $(Y \times Z)$ donnent les mêmes odds ratios que les tableaux partiels correspondants si $X$ et $Y$ sont conditionnellement indépendantes. A partir de ces résultats, on peut avoir une démarche pas à pas pour mesurer et interpréter les associations entre une variable réponse $Z$ (cpue) et des variables facteurs $X(d f)$ et $Y(\mathrm{~cm})$. Elle consiste à étudier les tableaux marginaux $(X \times Z)$ et $(Y \times Z)$ puis à vérifier sur le tableau $(X \times Y \times Z)$ que $X$ et $Y$ sont conditionnellement indépendantes.

Pour vérifier le bon ajustement de ces modèles aux observations, différentes statistiques de test sont possibles, nous utilisons la statistique de Wald et la statistique $G^{2}$ du rapport de vraisemblance qui permet de tester l'hypothèse que les effectifs espérés de la population satisfont à un modèle donné. Pour les grands échantillons, ces statistiques sont distribuées (on dit asymptotiquement distribuées) comme des $\chi^{2}$, la dimension de l'espace des paramètres correspondant à l'hypothèse alternative et à celle de l'hypothèse nulle. Si on ne fait pas d'hypothèse d'ordre sur les modalités des variables, les estimations des paramètres et les statistiques de test sont invariantes quel que soit l'ordre des catégories. Si on exploite le caractère ordonné des modalités des facteurs, on peut mettre en évidence des associations qui quantifient des tendances monotones uniformes par exemple (les cpue varient proportionnellement aux $\mathrm{cm}$ ) ou des ordonnancements stochastiques (les $d f$ ont des effets sur les cpue mais ces effets ne sont pas les mêmes sur les modalités de cpue). Ces derniers modèles sont plus faciles à interpréter que les modèles nominaux, ils peuvent être non saturés alors que leurs équivalents sur l'échelle nominale le sont, leurs tests sont plus puissants pour détecter certains types d'associations. 


\section{RÉSULTATS}

Pour étudier les associations entre les variables hydroclimatiques $(\mathrm{cm}$ et $d f$ ) et la variable de production (cpuce), nous avons analysé les Tableaux marginaux $2 a$ et $b$ puis le Tableau 1 . Les calculs ont été réalisés avec la procédure PROC CATMOD du logiciel SAS/STAT. Les procédures de tendance uniforme, d'ordonnancement stochastique et nominale sont données en annexe.

\section{Analyse des tableaux marginaux}

Les tableaux marginaux sont obtenus en sommant les comptages des cellules du Tableau 1 sur les modalités de $d f$ (Tableau $2 a$ ) et sur les modalités de $\mathrm{cm}$ (Tableau $2 b$ ). Les modèles loglinéaires nominaux les plus généraux appliqués aux données du Tableau $2 a\left(\log m_{j k}=\mu+\lambda_{j}^{Y}+\lambda_{k}^{Z}\right)$ et à celles du Tableau $2 b\left(\log m_{i k}=\mu+\lambda_{i}^{X}+\lambda_{k}^{Z}\right)$ sont des modèles d'indépendance. Les statistiques de test $G_{(\text {tabi. } 2 a)}^{2}=$ $23,88$ (Prob. $=0,005)$ et $G_{(\text {tabl. 2b) }}^{2}=25,17$ (Prob. $=$ $0,003)$ pour 9 degrés de liberté montrent que, dans chacun de ces cas, les variables ne peuvent être considérées comme indépendantes, les effectifs estimés sont très différents des effectifs observés, certains résidus ajustés (Haberman, 1973) sont élevés (Tableau $3 a$ et $b$ ). Pour le modèlc d'indépendance associé au Tableau $2 a$ les résidus ajustés sont définis par:

$$
r_{j k}=\frac{n_{j k}-\hat{m}_{j k}}{\left[\hat{m}_{j k}\left(1-p_{j+}\right)\left(1-p_{+k}\right)\right]^{1 / 2}}
$$

avec $\hat{m}_{j k}=n p_{j+} p_{+k}, p_{j+}$ et $p_{+k}$ étant les proportions marginales lignes et colonnes du Tableau $2 a$ (on a une formule analogue pour le modèle d'indépendance associé au Tableau $2 b$ ). Dans le Tableau $3 a$, les plus forts résidus positifs sont sur la première diagonale, les plus forts résidus négatifs sont sur la seconde diagonale. Cette structure particulière suggère que les variables $c m$ et cpue évoluent dans le même sens, il importe donc de modéliser cette tendance. Pour le Tableau $3 b$, on ne trouve pas de structure comparable des résidus, les évolutions conjointes de df et cpue. sont plus ératiques.

\section{Analyse de la liaison captures par unité d'effort-coefficient de marée}

On introduit dans le modèle un paramètre d'association entre cm et cpue en assignant, pour respecter l'ordre des modalités des variables, des scores croissants $\left\{u_{j}\right\}$ et $\left\{v_{k}\right\}$ aux lignes et aux colonnes du Tableau 2a. Le modèle peut alors s'écrire :

$$
\log m_{j k}=\mu+\lambda_{j}^{Y}+\lambda_{k}^{Z}+\beta u_{j} v_{k}
$$

Tableau 3. - Valeurs estimées des observations et résidus ajustés (entre parenthèses). Captures par unité d'effort: a) coefficient de marée; b) débit fluvial.

Estimated values of observations and adjusted residuals (in brackets). Catch per unit effort: a) tidal coefficient variables; b) rate of flow

\begin{tabular}{|c|c|c|c|c|}
\hline & \multicolumn{4}{|c|}{ Captures par unité d'effort } \\
\hline & Faible & Faible à Moyen & Moyen à Fort & it Fort \\
\hline \multicolumn{5}{|c|}{ a) Coefficient de marée } \\
\hline Faible & $\begin{array}{l}24,72 \\
(2,82)\end{array}$ & $\begin{array}{c}20,56 \\
(-0,16)\end{array}$ & $\begin{array}{c}20.56 \\
(-1.04)\end{array}$ & $\begin{array}{r}23,16 \\
(-1,73)\end{array}$ \\
\hline Faible à Moyen & $\begin{array}{c}23,33 \\
(0,18)\end{array}$ & $\begin{array}{c}19,40 \\
(2,86)\end{array}$ & $\begin{array}{c}19,40 \\
(-1.61)\end{array}$ & $\begin{array}{r}21,86 \\
(-1,39)\end{array}$ \\
\hline Moyen à Fort & $\begin{array}{c}22,50 \\
(-1,57)\end{array}$ & $\begin{array}{c}18,71 \\
(-2,03)\end{array}$ & $\begin{array}{c}18,71 \\
(1,90)\end{array}$ & $\begin{array}{l}21,08 \\
(1,72)\end{array}$ \\
\hline Fort & $\begin{array}{c}24,44 \\
(-1,51)\end{array}$ & $\begin{array}{c}20,33 \\
(-0,68)\end{array}$ & $\begin{array}{l}20,33 \\
(0,78)\end{array}$ & $\begin{array}{l}22,90 \\
(1,44)\end{array}$ \\
\hline $\begin{array}{l}\text { b) Débit fluvial } \\
\text { Faible }\end{array}$ & $\begin{array}{l}22,22 \\
(1,36)\end{array}$ & $\begin{array}{l}18,48 \\
(1,07)\end{array}$ & $\begin{array}{l}18,48 \\
(0,46)\end{array}$ & $\begin{array}{r}20,82 \\
(-2,86)\end{array}$ \\
\hline Faible à Moyen & $\begin{array}{c}25,(0) \\
(-1,37)\end{array}$ & $\begin{array}{c}20,79 \\
(-10,23)\end{array}$ & $\begin{array}{l}20,79 \\
(0,94)\end{array}$ & $\begin{array}{l}23,42 \\
(0,72)\end{array}$ \\
\hline Moyen à Fort & $\begin{array}{c}23,06 \\
(-2,83)\end{array}$ & $\begin{array}{c}19,17 \\
(-0,05)\end{array}$ & $\begin{array}{c}19,17 \\
(1,15)\end{array}$ & $\begin{array}{l}21,60) \\
(1,84)\end{array}$ \\
\hline Fort & $\begin{array}{l}24,72 \\
(2,83)\end{array}$ & $\begin{array}{c}20,56 \\
(-0,75)\end{array}$ & $\begin{array}{c}20,56 \\
(-2,50)\end{array}$ & $\begin{array}{l}23,16 \\
(0,24)\end{array}$ \\
\hline
\end{tabular}
variables.

il est intermédiaire entre le modèle d'indépendance $(\beta=0)$ et le modèle saturé $\left(\beta u_{j} v_{k}\right.$ est une forme particulière de $\left.\lambda_{j k}^{Y Z}\right)$, il ne fait perdre qu'un seul degré de liberté à la résiduelle. En utilisant les scores $\left\{u_{j}=j\right\}$ et $\left\{v_{k}=k\right\}$, espacés d'une unité, on remarque que pour deux lignes adjacentes $(j$ et $j+1)$ et deux colonnes adjacentes $(k$ et $k+1)$, $\log \left[\left(m_{j k} m_{j+1, k+1}\right) /\left(m_{j, k+1} m_{j+1, k}\right)\right]=\beta\left(u_{j+1}-\right.$ $\left.u_{j}\right)\left(v_{k+1}-v_{k}\right)=\beta$. Ainsi, $\theta_{j k}=\exp (\beta)$ est un odds ratio commun à toutes les paires de lignes et de colonnes adjacentes, il se réfère à des associations uniformes. En utilisant ces scores, on obtient : $G_{(\text {tabl. 2a) }}^{2}=10,90$ (Prob. $=0,208$ ) pour 8 degrés de liberté, $\hat{\beta}=0,152$ avec $\hat{\sigma}_{\hat{3}}=0,04$ pour écart-type asymptotique. La valeur positive de $\hat{\beta}$ indique que les cpuc tendent à être d'autant plus grandes que les valeurs des modalités de $\mathrm{cm}$ sont plus grandes. L'estimation du odds ratio uniforme $(\exp (0,152)=1,16)$ indique que par rapport à la catégorie $k$, la cpue de la catégorie $k+1$ a augmenté d'un facteur multiplicatif 1,16 pour chaque changement croissant de catégorie pour cm. En considérant les cellules des coins extrêmes du Tableau $2 a$, le odds ratio estimé pour une forte cpue est exp $[0,152(4-1)(4-1)]=3,93$ fois plus important pour la catégorie "Fort" que pour la catégorie "Faible" de $\mathrm{cm}$. Un intervalle de confiance à $95 \%$ pour exp $(\beta)$ est $\exp (0,152 \pm 1,96 \times 0,04)$, soit $(1,08 ; 1,26)$. 
Le modèle (4) a structurellement la forme du logarithme de la densité d'une distribution binormale. Ce modèle tend à donner un bon ajustement quand la distribution continue sous-jacente est approximativement binormale. Pour des scores standardisés, $\beta$ est comparable à $\rho /\left(1-\rho^{2}\right)$, où $\rho$ est le coefficient de corrélation (Agresti, 1990). Si les associations sont faibles $\beta \simeq \rho$. L'analyse avec les scores standardisés donne : $G_{(\text {tabl. 2a) }}^{2}=11,77$ (Prob. $=0,162$ ) pour 8 degrés de liberté, $\hat{\beta}=0,196$ avec $\hat{\sigma}_{\hat{\beta}}=0,06$ pour écart-type asymptotique. S'il y a une distribution sous-jacente binormale, la corrélation estimée est $\hat{\rho}=0,189$. Ainsi, le modèle d'association uniforme met en évidence une association positive relativement faible.

\section{Analyse de la liaison captures par unité d'effort-débit fluvial}

Nous avons vu, dans l'analyse des résidus, que les évolutions conjointes des df et des cpue ne mettaient pas en évidence l'existence éventuelle d'une tendance (Tableau $2 b$ ). Le modèle précédent n'est donc pas adapté, on ne peut plus considérer qu'il y a une relation d'ordre sur $d f$ d'une part et sur cpue d'autre part. Puisque nous cherchons à expliquer l'importance de la variable réponse cpue par la variable facteur $d f$, nous ne prendrons en compte que la relation d'ordre sur les cpue. Dans ce cas, le modèle s'écrit :

$$
\log m_{i k}=\mu+\lambda_{i}^{X}+\lambda_{k}^{Z}+\mu_{i} v_{k}
$$

les $\left\{v_{k}\right\}$ sont des scores et les $\left\{\mu_{i}\right\}$ sont des effets lignes. Le modèle $(5)$ a $(I-1)$ paramètres de plus que le modèle d'indépendance dans lequel tous les $\mu_{i}$ seraient égaux.

En utilisant les scores $\left\{v_{k}=k\right\}$, on obtient : $G_{(\text {tabl. } 2 b)}^{2}=9,55$ (Prob. $\left.=0,145\right)$ pour 6 degrés de liberté. Pour les lignes $h$ et $i$ et les colonnes adjacentes $k$ et $k+1$, le logarithme des odds ratios $\left(\log \left[\left(m_{h k} m_{i, k+1}\right) /\left(m_{h, k+1} m_{i k}\right)\right]=\mu_{i}-\mu_{h}\right)$ est identique pour toutes les $K-1$ paires de colonnes adjacentes. Les $\left\{\mu_{i}-\mu_{h}\right\}$ décrivent les différences entre lignes. Quand $\mu_{i}=\mu_{h}$, les lignes $i$ et $h$ ont des distributions identiques. Si $\mu_{i}>\mu_{h}$, les cpue sont stochastiquement plus élevées dans la ligne $i$ que dans la ligne $h$. Avec les contraintes d'identification des paramètres définies précédemment, on trouve $\mu_{1}=$ $-0,221, \mu_{2}=0,116, \mu_{3}=0,252$ et $\mu_{4}=-0,147$. On en déduit que les cpue sont stochastiquement plus importantes pour la catégorie "Moyen à Fort" que pour la catégorie "Faible à Moyen". Des conclusions comparables peuvent être tirées en considérant les autres couples $\mu_{i}$ et $\mu_{h}$.

La première analyse montre que le niveau des captures par unité d'effort augmente proportionnellement à la valeur du coefficient de marée. La deuxième analyse met en évidence un mécanisme d'action différent, les débits fluviaux "Faible" ou "Fort" sont peu propices à des cpuc importantes alors que les débits fluviaux "Faible à Moyen" et "Moyen à Fort" sont plus favorables, ces relations n'étant que stochastiquement vérifiées. Ces conclusions étant déduites de deux analyses séparées, rien ne peut être dit sur les interactions éventuelles entre $c m$ et $d f$, elles doivent donc être complétées par une analyse globale du Tableau 1.

\section{Analyse globale}

Considérons maintenant le modèle

$$
\log m_{i j k}=\mu+\lambda_{i}^{X}+\lambda_{j}^{Y}+\lambda_{k}^{Z}+\lambda_{i j}^{X Y}+\lambda_{i k}^{X Z}+\lambda_{j k}^{Y Z}
$$

Les résultats de cette analyse sont donnés dans le Tableau 4, ils montrent la bonne adéquation des données au modèle, $G_{\text {résid u }}^{2}=26,16$ (Prob. $=0,510$ ) pour 27 degrés de liberté précise que les résidus sont peu importants. Conditionnellement aux cpue, $\mathrm{cm}$ et $d f$ sont indépendantes, la statistique de Wald est égale à 8,51 (Prob. $=0,484$ ) pour 9 degrés de liberté, seules les interactions $c m \times$ cpue et $d f \times c p u e$ sont significatives, les statistiques de Wald étant respectivement égales à $24,66($ Prob. $=0,003)$ et 23,51 $($ Prob. $=0,005)$ pour 9 degrés de liberté. D'après les résultats du paragraphe sur les modèles, on est en droit d'accepter que les variables $c m$ et $c p u c$ d'une part, $d f$ et cpue d'autre part sont liées par les relations décrites dans l'étude des tableaux marginaux.

Pour décrire ces données par un modèle plus parcimonieux, on structure l'interaction $c m \times c p u c$ en assignant des scores croissants $\left\{u_{j}\right\}$ à $c r n$ et $\left\{v_{k}\right\}$ à cpue. Ce nouveau modèle s'écrit $: \log m_{i j k}=\mu+\lambda_{i}^{X}+\lambda_{j}^{Y}+$ $\lambda_{k}^{Z}+\lambda_{i j}^{X Y}+\lambda_{i k}^{X Z}+\beta u_{j} v_{k}$ (le terme $\lambda_{j k}^{Y Z}$ a été remplacé par $\left.\beta u_{j} v_{k}\right)$. Les odds ratios conditionnels satisfont à $\log \left[\left(m_{i j k} m_{i, j+1, k+1}\right) /\left(m_{i, j, k+1} m_{i, j+1, k}\right)\right]=$ $\beta\left(u_{i+1}-u_{i}\right)\left(v_{j+1}-v_{j}\right)=\beta(1 \leq j \leq J-1$, $1 \leq k \leq K-1)$ pour tout $i, G_{\text {(tabl. 1) }}^{2}=38,46$ (Prob. $=0,316$ ) pour 35 degrés de liberté, $\hat{\beta}=0,155$ avec $\hat{\sigma}_{\hat{\beta}}=0,04$ pour écart-type asymptotique. Les estimations de $\hat{\beta}$ et de $\hat{\sigma}_{\hat{\beta}}$ sont identiques à celles

Tableau 4. - Tableau d'analyse de variance : captures par unité d'effort (cpue), coefficient de marée ( $\mathrm{cm}$ ) et débit fluvial (df), degrés de liberté (d.d.1.).

Analysis of variance table: catch per unit effort (cpue), tidal coefficient $(\mathrm{cm})$, rate of flow $(d f)$ variables, degrees of freedom (d.d.l.)..

\begin{tabular}{crcc}
\hline Source & d.d.l. & $\begin{array}{c}\text { Statistique de } \\
\text { Wald }\end{array}$ & Probabilité \\
\hline cpue & 3 & 0,97 & 0,810 \\
cm & 3 & 0,69 & 0,875 \\
df & 3 & 1,35 & 0,717 \\
cm $\times$ cpue & 9 & 24,66 & 0,003 \\
df $\times$ cpue & 9 & 23,51 & 0,005 \\
cm $\times$ df & 9 & 8,51 & 0,484 \\
Résidu & 27 & 26,16 & 0,510 \\
\hline
\end{tabular}


trouvées précédemment $\left(\hat{\beta}=0,152, \hat{\sigma}_{\hat{\beta}}=0,04\right)$. Ce dernier modèle est tout à fait satisfaisant, on ne peut plus le simplifier car, comme nous l'avons vu, les relations entre $d f$ et crutue ne sont que stochastiquement vérifiécs.

\section{DISCUSSION}

Sur les données analysées, on aurait pu raisonner conditionnellement à $\mathrm{cm}$ et $d f$ et utiliser le modèle logit généralisé si on ne voulait pas tenir compte de l'ordre sur la variable réponse (Prouzet et al., 1994) ou des modèles logit adjacent ou logit cumulé pour prendre en compte l'ordre sur les cpue. Appliqués aux aloses, tous ces modèles donnent des résultats comparables, ils suggèrent l'impact des variations conjuguées du débit fluvial et du coefficient de marée sur l'abondance des captures journalières, le modèle (6) est d'ailleurs identique au modèle logit généralisé (McCullagh and Nelder, 1989). Une autre approche possible, essentiellement exploratoire, serait l'analyse des correspondances du tableau de contingence croisant $(d f \times c r n)$ avec cprle pour visualiser les profils lignes (Prouzet et al., 1994) et tester approximativement l'influence de $d f$ et cm sur la dispersion de ces profils (Tenenhaus et al., 1993). A toutes ces analyses, nous préférons celle basée sur la modélisation des interactions dans le modèle loglinéaire car elle permet de quantifier les liens entre deux variables. Ces liaisons n'ćtant pas identifiées par des expressions analytiques exactes, le choix a priori de scores régulièrement espacés semble raisonnable et de plus ils sont facilement interprétables. Si des scores régulièrement espacés ne s'imposaient pas de façon naturelle, il serait nécessaire de compléter ces analyses par une étude de sensibilité en éprouvant plusieurs ensembles de scores et en vérifiant si les conclusions diffèrent fondamentalement. D'un point de vue général les tableaux de contingences sont des représentations simplifiées d'observations continues, les catégories sont choisics par l'expérimentateur, clles dépendent de la distribution des variables continues sous-jacentes et des objectifs assignés à l'étude. Les scores doivent être le reflet de la construction du tableau de contingence ct de son utilisation. Le problème traité en est un exemple, la modélisation des interactions à partir de scores également espacés est ici un choix admissible cu égard à la construction des catégories.

La mise en cuvre de ces modèles est simple ( $c f$. annexe), elle peut se résumer en disant qu'elle débute par l'utilisation du modèle loglinéaire nominal Ic plus général qui s'ajuste bien aux données et qu'elle est poursuivic en recherchant lc modèle le plus parcimonieux, les paramètres d'interaction étant remplacés par des termes structurés tenant compte de l'ordre sur les modalités des variables.

L'utilisation de ces modèles en hydrobiologie peut être généraliséc à l'étude des interactions entre plusieurs variables dont l'une est à expliquer. Les exemples sont nombreux : variation des densités en fonction des caractéristiques du milieu; fréquence d'infestation d'un parasite en fonction des saisons, du milieu et des caractéristiques de l'hôte, etc. Plus généralement, ils permettent de compléter les résultats obtenus par des analyses descriptives multivariées (Tenenhaus et al., 1993) par la modélisation de la loi de probabilité d'une variable $y$ en fonction des variables explicatives et de décrire l'influence de celleci sur la loi de probabilité de $y$. Ainsi que le notent ces auteurs, le modèle linéaire généralisé permet de valider statistiquement des résultats que l'analyse des correspondances ne fait que suggérer.

\section{ANNEXE}

Les modèles utilisés sont ajustés en spécifiant la matrice du modèle d'un modèle logit généralisé.

\section{Iiaison coefficient de marée-captures par unité d'effort}

Pour le modèle d'association uniforme, la matrice du modèle est de dimension $12 \times 4$ puisqu'il y a trois logits $\left(\log \left(m_{j k} / m_{j 4}\right)\right)$ dans chaque ligne du Tableau $2 a$ et quatre paramètres à estimer. Les trois premiers paramètres d'une ligne se réferent aux interceptions des trois logits. En utilisant les scores $u_{j}=j$ et $v_{k}=k$, le coefficient de $\beta$ dans le modèle logit est $u_{j}\left(v_{k}-v_{4}\right)$.

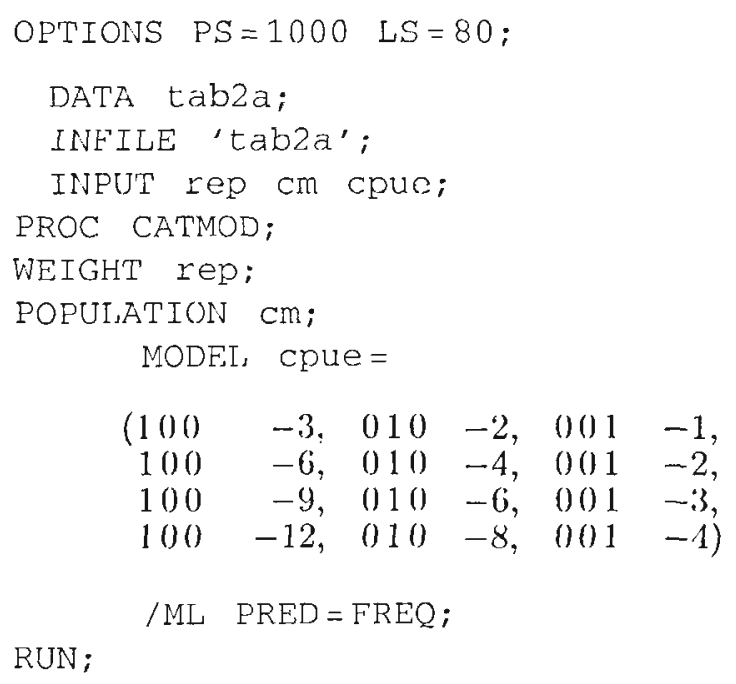

\section{Liaison débit fluvial et captures par unité d'effort}

Le modèle d'association stochastique utilise la contrainte $\mu_{4}=-\left(\mu_{1}+\mu_{2}+\mu_{3}\right)$, les quatrième, cinquième et sixième éléments de chaque ligne sont les coefficients $\left(v_{k}-v_{4}\right)$ de $\mu_{1}, \mu_{2}$ et $\mu_{3}$ du modèle logit généralisé $\left(\log \left(m_{i k} / m_{i 4}\right)\right)$. 


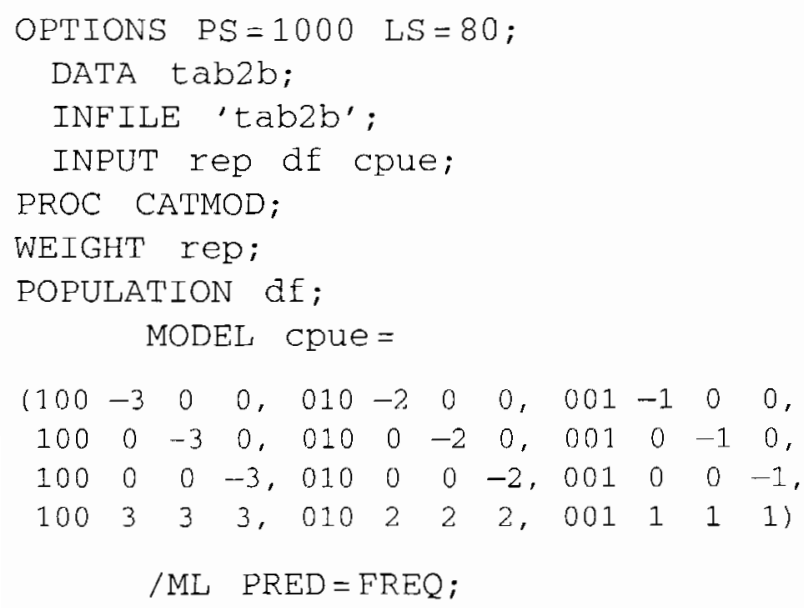

RUN ;

\section{Liaison coefincient ca marée-débit fuvial- captures par unité d'efíert}

Pour le modèle loglinéaire nominal le plus général on utilise LOGLIN.

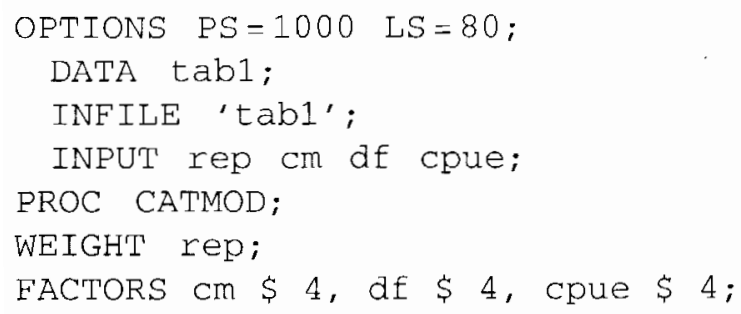

MODEL

PRED = FREQ ;

LOGLIN cpue $\mathrm{cm}$ df cpue*cm cpue*df $\mathrm{cm} * \mathrm{df}$;

RUN;

\section{RÉTERTICES}

Agresti A. 1990. Categorical Data Analysis. John Wiley \& Sons. New York, 558p.

Bishop Y. V. V. 1971. Effects of collapsing multidimensional contingency tables. Biometrics 27, 545-562.

Bishop Y. V. V., S. E. Fiengerg, P. Holland 1975. Discrete Multivariate Analysis: theory and practice, MIT Press, Cambridge, Mass.

Fierberg S. E., 1900. The analysis of cross classified categorical data, 2nd ed. MIT Press, Cambridge, Mass.

Haberman S. J. 1973. The analysis of residuals in crossclassification tables. Biometrics 2), 205-220.

McCullagh P., J. A. Nelder 1989. Generalized Linear Models. Monographs on statistics and applied probability. Chapman and Hall, London.

Prouzet P., J. P. Martinet, J. Badia 1994. Caractérisation biologique et variations des captures de la grande alose (Alosa alosa) par unité d'effort sur le fleuve Adour, (Pyrénées Atlantiques, France). Aquat. Living Resour. 7, $1-10$.

SAS Institute 1990. SAS/STAT User's guide. Version 6, Fourth Edition.

Tenenhaus M., Y. Le Roux, C. Guimard, P. L. Gonzalez 1993. Moḋ̀le linéaire généralisé et analyse des correspondances. Rev. Stat. Appl. 41, 59-86. 